\title{
Daily Patterns of Optimal Producer and Scrounger Use under Predation Hazard: A State-Dependent Dynamic Game Analysis
}

\author{
Zoltán Barta $^{1, *}$ and Luc-Alain Giraldeau ${ }^{2, \dagger}$ \\ 1. Behavioural Ecology Research Group, Department of \\ Evolutionary Zoology, Kossuth University, Debrecen H-4010, \\ Hungary; \\ 2. Department of Biology, Concordia University, 1455 ouest \\ Boulevard de Maisonneuve, Montréal, Québec H3G 1M8, Canada \\ Submitted March 15, 1999; Accepted October 13, 1999
}

\begin{abstract}
Feeding in groups often gives rise to joining: feeding from other's discoveries. The joining decision has been modeled as a producer-scrounger game where the producer strategy consists of searching for one's food and the scrounger strategy consists of searching for food discovered by others. Previous models revealed that the evolutionarily stable proportion of scrounging mostly depends on the fraction of each food patch available only to its producer. These early models are static and state independent and are therefore unable to explore whether the time of day, the animal's state, and the degree of predation hazard influence an individual's decision of whether to use the producer or scrounger strategy. To investigate these issues, we developed a state-dependent dynamic producer-scrounger game model. The model predicts that, early in the day, low reserves promote a preference for the scrounger strategy, while the same condition late in the day favors the use of the producer strategy. Under rich and clumped food, the availability of scrounging can improve the daily survival of any average group member. The model suggests only weak effects of predation hazard on the use of scrounging. Future developments should consider the effects of dominance asymmetries and allowing foragers a choice between foraging alone or in a group harboring an evolutionarily stable frequency of scrounger.
\end{abstract}

Keywords: social foraging, producer-scrounger game, state-dependent dynamic game.

Most animals' behavior follows a more or less regular daily pattern (routine). Recent theoretical investigations of

\footnotetext{
* E-mail: zbarta@dragon.klte.hu.

† E-mail: giraluc@vax2.concordia.ca.
}

Am. Nat. 2000. Vol. 155, pp. 570-582. (c) 2000 by The University of Chicago. 0003-0147/2000/15504-0011\$03.00. All rights reserved. state-dependent dynamic models (reviewed by Cuthill and Houston 1997; Houston and McNamara 1999) identify several innately associated trade-offs among the day-night cycle, gaining/consuming energy and avoiding predation, which can generate these routines even in the absence of varying external variables (e.g., food availability or temperature). Night (the nonforaging period), for instance, notably reduces the animal's energy reserves, which can only be replenished during daylight. Foraging increases energy reserves at the expense of increased predation hazard, while resting offers more safety against predators but consumes energy (Houston et al. 1993). As a consequence of these trade-offs, different behavioral actions are optimal during different parts of the day, leading to the emergence of daily routines. As a result of recent theoretical and empirical investigations (e.g., McNamara et al. 1994; Witter et al. 1994; Cuthill and Houston 1997; Dall and Witter 1998; Houston and McNamara 1999; Van der Veen, in press $a$, in press $b$ ), a lot is known both about the solitary animals' daily routines and about the factors influencing them but the analysis of daily routines of animals taking part in social foraging interactions is still missing.

The exploitation of companions' search effort is one of the most common forms of social-foraging interactions that occur both within (Giraldeau and Beauchamp 1999) and between species (Sibly 1984; Barnard and Thompson 1985). In ground-feeding passerines, for instance, some individuals actively search for food patches while others appear to wait for them to find a patch, rushing in to secure a share when they do (Barnard and Sibly 1981; Giraldeau et al. 1994).

In order to account for the coexistence of feeding both through finding and joining within a single-species group, the phenomenon has been modeled as a static, alternativeoption, $n$-person evolutionary game (Barnard and Sibly 1981; Maynard Smith 1982; Parker 1984). The game assumes that individuals have access to two mutually exclusive foraging strategies: producer, which consists of searching for food, or scrounger, which consists of searching for 
individuals that have found food. Individuals can be pure producer, pure scrounger, or, more commonly, they can alternate between strategies. It is important to keep in mind, however, that individuals that alternate can only use one or the other strategy at any one time (Giraldeau and Beauchamp 1999). When scrounging is rare in the group, the scrounger strategy is assumed to do better than its producer alternative because it can take advantage of the many exploitable food patches that are made available by the numerous individuals engaged in the producer strategy. However, the reverse holds when scrounging becomes common; now the number of available food patches decreases as a consequence of the decreasing number of individuals in the producer strategy while at the same time the number of individuals competing within the scrounger strategy is greater (Barnard and Sibly 1981). In a symmetric game involving phenotypically equivalent players, this strong negative frequency dependence often leads to an evolutionarily stable strategy (ESS) that contains both foraging options occurring at a frequency where payoffs obtained by each are equal: a mixed ESS (Maynard Smith 1982; Parker 1984). Using this game-theoretic framework, a number of producer-scrounger foraging models, whether based on food intake maximization (Vickery et al. 1991; Ranta et al. 1996) or starvation risk minimization (Caraco and Giraldeau 1991), have concluded that the equilibrium frequency of scrounger within any group depends strongly on the fraction of each food patch available only to its producer, that is, the finder's share and the group size. Increasing the finder's share leads to a decreasing equilibrium proportion of scroungers by decreasing the scroungers' gain from a patch. On the other hand, increasing group size raises the stable proportion of individuals in the scrounger strategy because larger groups mean a greater number of exploitable individuals in the producer strategy.

Some empirical studies (e.g., Giraldeau et al. 1990, 1994; Koops and Giraldeau 1996) support the previous producer-scrounger models' predictions. The qualitative successes of these static models suggest that a dynamic, statedependent version would be useful in exploring the conditions under which we expect optimal daily routines of producer and scrounger use. Consequently, we develop here a state-dependent dynamic producer-scrounger game. The state-dependent dynamic game approach compares different actions through a common currency: survival in our case (McNamara and Houston 1986). This common currency allows us to ask how the use of scrounging can influence the individuals' survival probability as well as explore any potential effects of other nonforaging constraints, such as predation hazards. As a consequence, a dynamic model can shed light on the role of the scrounger strategy in the dynamics of populations (McNamara and Houston 1987).

\section{The Model}

We considered small seed-eating birds surviving several winter days and nights. During the day, the birds can choose to rest or to forage, in which case they can choose to play either producer or scrounger. During the night, all birds rest. The birds' energy reserves decline as the result of the metabolic cost. To survive the night, the birds need a large, sometimes randomly fluctuating energy reserve. A bird can be captured by a predator only while it forages. Predation hazard is mass dependent and increases with a bird's energy reserves (Witter and Cuthill 1993; Witter et al. 1994). The birds must therefore keep their reserves at a reasonable level (for details of the model, see the appendix; McNamara et al. 1994).

The birds' energy reserves can increase only through foraging activity. We assume that the birds always feed in a flock of $N$ individuals. This means that, considering a large population, a bird is always able to find a foraging group of size $N-1$ in which to forage, independently of the proportion of resting birds in the population; that is, resting birds are not considered to be part of the foraging flock. This would be a reasonable assumption in the case of a large bird population living and resting at a focal point (e.g., communal roost) surrounded by fields where they feed in small flocks. Foraging involves the use of either producer or scrounger for the duration of the time interval. So, the proportion of scroungers $\left(p_{\mathrm{S}}\right)$ remains constant during an interval. The number of scroungers $\left(N_{\mathrm{S}}\right)$ in the flock is $N p_{S}$, while the number of producers $\left(N_{\mathrm{P}}\right)$ is $N\left(1-p_{\mathrm{S}}\right)$.

We presume that producers search for food independently of each other and a producer can only feed from patches it uncovers itself. Scroungers, instead, can only feed from patches uncovered by producers. We also assume that patch exploitation time is negligible compared to search time and that individuals are competitively equal. It follows that all scroungers in the flock $\left(N_{\mathrm{s}}\right)$ obtain the same share of each discovered patch and that each of the producers' patch findings are detected by all those playing scrounger. Upon finding a patch, a producer first consumes some food while alone (the finder's advantage, $a$; Vickery et al. 1991) and then shares the remainder food with the arriving scroungers. The finder's share (FS) is defined as the proportion of food consumed by the producer to the total amount of food in the patch. The FS may depend on the handling time of a food item (the larger the handling time, the smaller the FS) or on the average distance of foragers in the flock (the larger the distance, the larger the FS). In the model, we consider the FS as an ecological constraint over which the foragers have no control (for further details, see the appendix).

When there is more than one individual playing pro- 


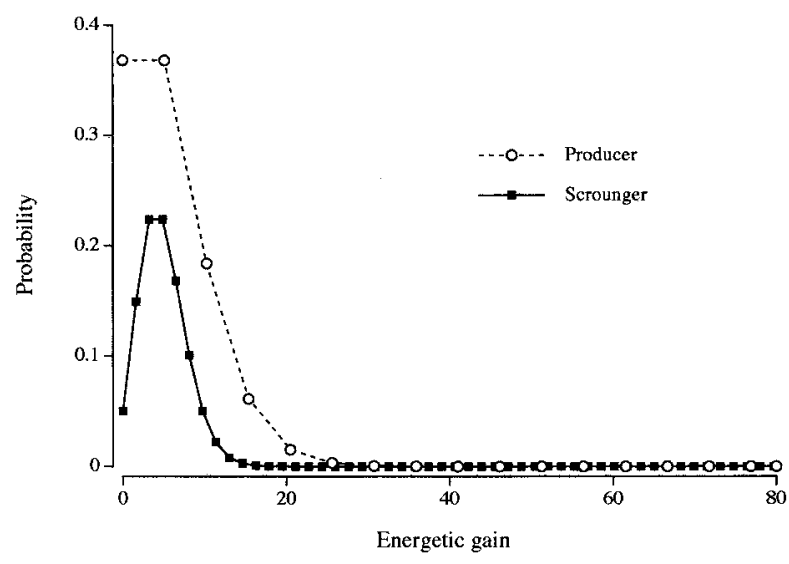

Figure 1: Probability distribution of energetic gain of producers and scroungers during an interval. $\left(N_{\mathrm{p}}=3 ; N_{\mathrm{s}}=2\right.$; other parameters as baseline).

ducer in the group, the probability of eating nothing during an interval is higher for individuals in the producer strategy than those in the scrounger (fig. 1). On the other hand, the producer has a higher chance of obtaining large amounts of food because, when FS $>0$, a producer always gains more from a patch than the scroungers (fig. 1). On the basis of these properties, the producer is said to be risk prone, while the scrounger is risk averse (Caraco and Giraldeau 1991; Koops and Giraldeau 1996).

A policy is a decision rule that specifies which action to choose as a function of time of day and energy reserves (Houston and McNamara 1988b). State-dependent optimal policies are usually found by dynamic programming (McNamara and Houston 1986; Houston et al. 1988; Mangel and Clark 1988), but in the case of producer-scrounger games, we could not proceed this way because an individual's gain is frequency dependent. Specifically, an individual's gain, here, depends on the proportion playing scrounger. Therefore, we followed the iteration procedure proposed by Houston and McNamara (1987, 1988a) and modified by McNamara et al. (1997) to determine the evolutionarily stable dynamic policy. To calculate the optimal policy, we used the "errors in decision making" approach (McNamara et al. 1997), which makes the biologically relevant assumption that animals may make mistakes during the decision process. The probability of errors, however, depends on their costs so that actions that seriously decrease the animal's reproductive value compared to the best available action are chosen very rarely, while mistakes with trivial consequences can be made more often. So, if two actions differ only slightly in their effects on the animal's reproductive value, the individual will choose them with more or less equal probability. Intro- ducing errors in decision making eliminates most of the problems with the above iterative method (for further details, see appendix; McNamara et al. 1997).

The key parameter of this approach is the extent of possible error $(\delta)$. When $\delta$ is 0 , the animals make decisions without error, whereas increasing $\delta$ means animals are increasingly unable to differentiate actions that have similar consequences. Preliminary calculations showed that our foraging model was quite sensitive to the value of $\delta$ (fig. 2), so we chose the smallest possible value of $\delta$ $(=0.001)$ that allowed us to find a stable policy in most cases to minimize the effect of $\delta$.

\section{Results}

At first, we show the results for a baseline case (table 1). The baseline is chosen so that its results represent general patterns found across a wide range of parameter values. After that we investigate the effects of some important (from the point of view of social foraging) parameters of the model by altering their values one at a time.

\section{The Baseline Case}

The evolutionarily stable dynamic policy depends on both the time of day and the energy reserves (fig. $3 A$ ). It is always optimal to rest when reserves are very high, and this is true throughout the day. In the first part of the day, a decrease in energy reserves leads to what we call a probabilistic region. In this region, as the reserves decline, the probability of resting also declines, the likelihood of playing scrounger increases while the occurrence of producer first increases and then decreases. At reserve levels below

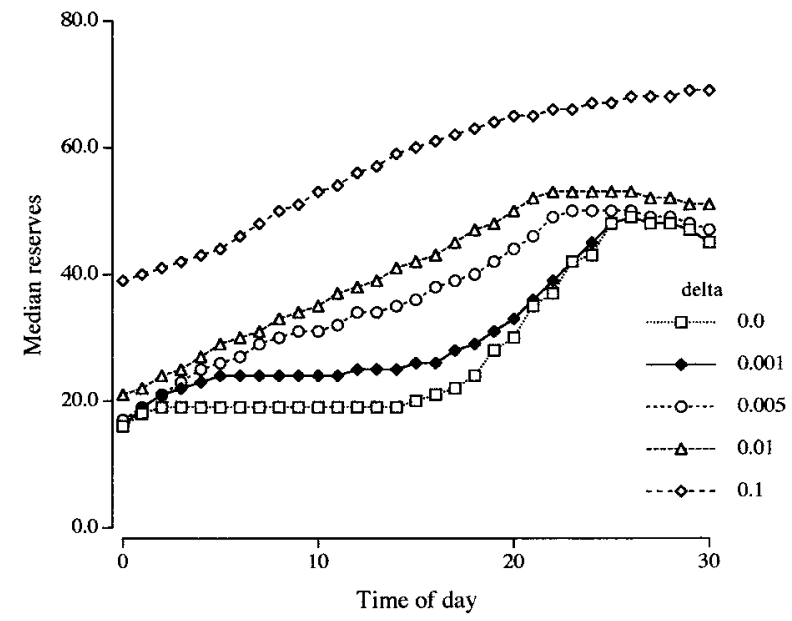

Figure 2: Effect of $\delta$ (the extent of error; McNamara et al. 1997) on the trajectories of reserves along the day (baseline except FS $=1.0$ ). 
Table 1: Parameters of the model

\begin{tabular}{|c|c|c|}
\hline Parameter & Meaning & Baseline \\
\hline \multicolumn{3}{|l|}{ Group: } \\
\hline$N$ & Group size & 5 \\
\hline \multicolumn{3}{|l|}{ Foraging: } \\
\hline Y & Amount of food in a patch & 10.0 \\
\hline$\lambda$ & Patch-finding rate per interval for producers & 1.0 \\
\hline FS & Finder's share & .35 \\
\hline \multicolumn{3}{|c|}{ State variable: } \\
\hline$x(t)$ & Energy reserves, $0 \leq x(t) \leq x_{\max }$. Animal dies if $x=0$ & \\
\hline$x_{\max }$ & Upper limit of reserves & 80.0 \\
\hline \multicolumn{3}{|l|}{ Time: } \\
\hline$T$ & Length of day & 30 \\
\hline$D$ & Number of days & 5 \\
\hline \multicolumn{3}{|l|}{ Metabolism: } \\
\hline$d 0_{\mathrm{r}}$ & Daytime resting metabolic expenditure per time interval at reserves $x=0$ & 1.0 \\
\hline$d 2_{\mathrm{r}}$ & Rate of increase of resting metabolism expenditure with reserves & .000125 \\
\hline$d 0_{\mathrm{p}}, d 0_{\mathrm{s}}$ & Foraging metabolic expenditure per time interval at reserves $x=0$ & 2.0 \\
\hline$d 2_{\mathrm{p}}, d 2_{\mathrm{s}}$ & Rate of increase of foraging metabolism expenditure with reserves & .00025 \\
\hline$\mu_{\text {night }}$ & Mean overnight expenditure & 30.0 \\
\hline$\sigma_{\text {night }}$ & Standard deviation of overnight expenditure & 3.0 \\
\hline \multicolumn{3}{|l|}{ Predation: } \\
\hline$m 0_{\mathrm{p}}, m 0_{\mathrm{s}}$ & Predation hazard per interval for a foraging animal at reserves $x=0$ & .0002 \\
\hline$m 2_{\mathrm{p}}, m 2_{\mathrm{s}}$ & Rate of increase of predation hazard with squared reserves & .00001 \\
\hline \multicolumn{3}{|c|}{$\begin{array}{l}\text { Finding evolutionarily } \\
\text { stable dynamic } \\
\text { policy: }\end{array}$} \\
\hline$\delta$ & Extent of error in decision making & .001 \\
\hline$r f$ & Replacement factor & .2 \\
\hline$i_{\max }$ & Maximal number of iterations & 5,000 \\
\hline$D_{\min }$ & Iteration stops if difference between subsequent policies smaller than $D_{\min }$ & .00001 \\
\hline
\end{tabular}

those generating the probabilistic region, the optimal decision is to play scrounger only. The probabilistic region occurs for low energy reserves early in the day, but as time of day increases, it occurs at gradually increasing energy levels, peaking some time in the afternoon. After this peak, the optimal pattern of behavior changes. The probabilistic range disappears so that from high to low reserves, the optimal strategy now goes from resting, to playing scrounger, to playing producer. In groups of birds following the optimal policy, the daily pattern of playing scrounger is $\mathrm{U}$-shaped. The proportion of scroungers peaks around dawn and dusk and is lowest during midday (fig. 3B). Furthermore, the energy reserves of individuals playing scrounger are lower than the reserves of individuals playing producer for the first part of the day, but the reverse is true later in the day (fig. $3 C$ ). These patterns can be explained on the basis of differences in the risk sensitive properties of producer and scrounger, and they hold for wide range of parameter values.
Finder's Share

We investigated the effect of FS by systematically varying its value from 0 to 1 , keeping all other parameters constant. Increasing FS in our model leads to a decrease in the daily average proportion of scrounging (fig. 4A). This decrease is in agreement with predictions of earlier static models (Caraco and Giraldeau 1991; Vickery et al. 1991). However, the dynamic model predicts more scroungers at larger FS than the static rate-maximizing model.

Moreover, the FS also greatly influences the daily pattern of stable proportion of scrounging (fig. $4 B$ ). At small FS, the proportion of scrounger remains constant over the day. Under this condition, the risk-sensitive properties do not differ much for the two strategies; therefore, it makes sense not to use them differently along the course of the day. As the FS, and so the differences between the strategies, increases, the daily pattern of scrounging becomes increasingly U-shaped, with peak scrounger frequencies at dawn and dusk and lower frequencies around midday, 


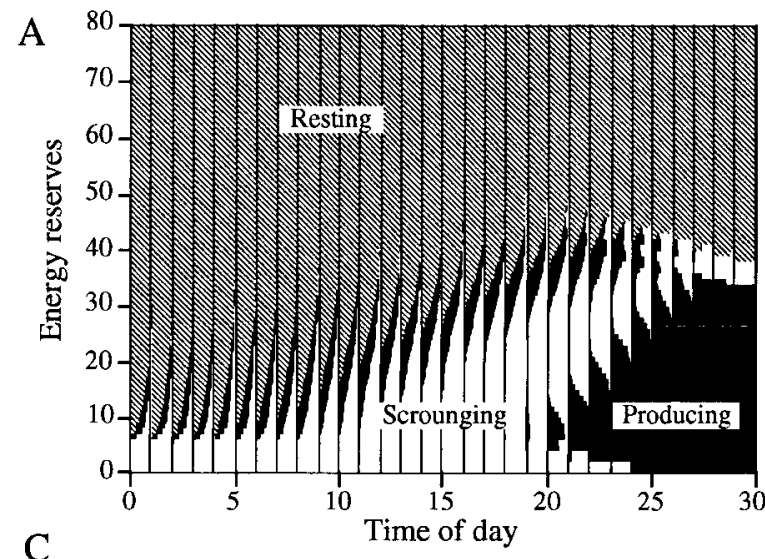

C

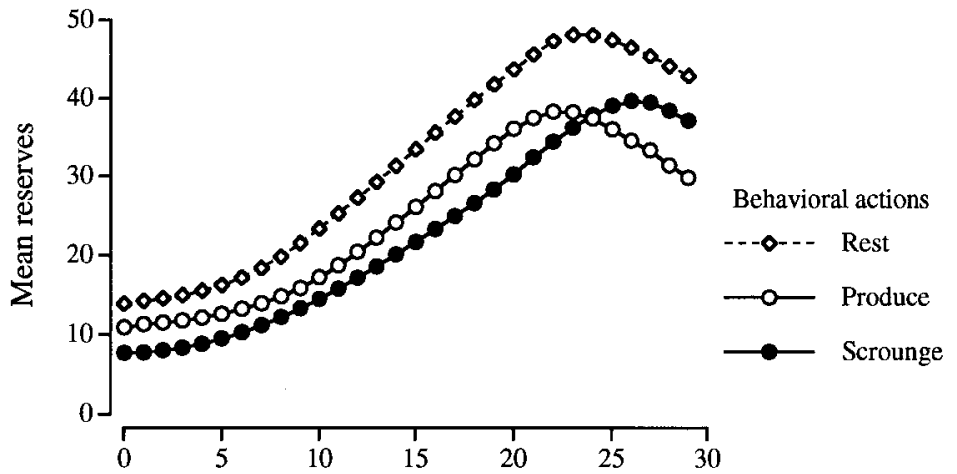

B

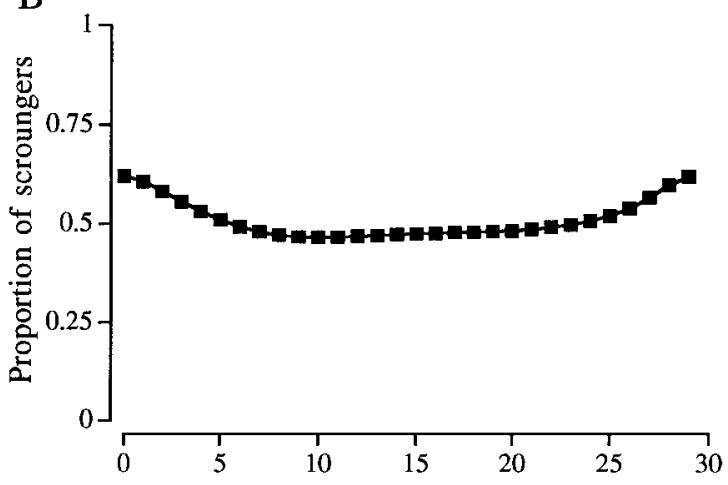

Time of day

Time of day

Figure 3: Baseline case. A, Evolutionarily stable dynamic policy that shows what action is optimal as the function of reserves and time of day. $B$, Stable proportion of scroungers in the foraging group along the day. $C$, Mean reserves of individuals performing different behavioral action.

much as in the baseline case. As the FS approaches 1.0, where no profitable patch sharing (i.e., use of scrounging) is possible, the daily scrounger pattern becomes flat again but at a very low frequency (the frequency of the scrounger strategy remains above 0 as a consequence of the "errors in decision making" approach that is required by our modeling).

The FS also influences the daily foraging routines (fig. $4 C)$. At low FS, the birds spend most of their time foraging. As FS increases, birds narrow their declining foraging activity to early morning and midafternoon. The decreasing foraging activity results from the pattern of food sharing between producer and scrounger. High FS means a low stable proportion of scroungers, which increases the gain of an average group member by decreasing the number of competing individuals in a patch. As a consequence of increased energetic gain, the individuals can spend less time foraging during the day, and they are still able to collect enough food to survive the night (McNamara et al. 1994). Minimizing time spent foraging is important because only foraging individuals can be caught by predators. As a result, the probability of daily survival increased with FS. However, this should not always be the case, as we see below. The effects of FS manipulation on the foraging activity are analogous to those of changing food abundance in other dynamic model studies (e.g., McNamara et al. 1987, 1994).

Food

We manipulate food in two ways. First, we change patchiness by varying both the patch-finding rate and the amount of food in a patch so that their product (i.e., the average gain) remains constant. Varying patchiness does not change the average daily stable proportion of scrounging. Increasing patchiness decreases the probability of survival at most values of the FS (fig. 5). This effect may come from the increased stochasticity in food supply (McNamara and Houston 1987). When food is evenly distributed or moderately aggregated, daily survival decreases with 


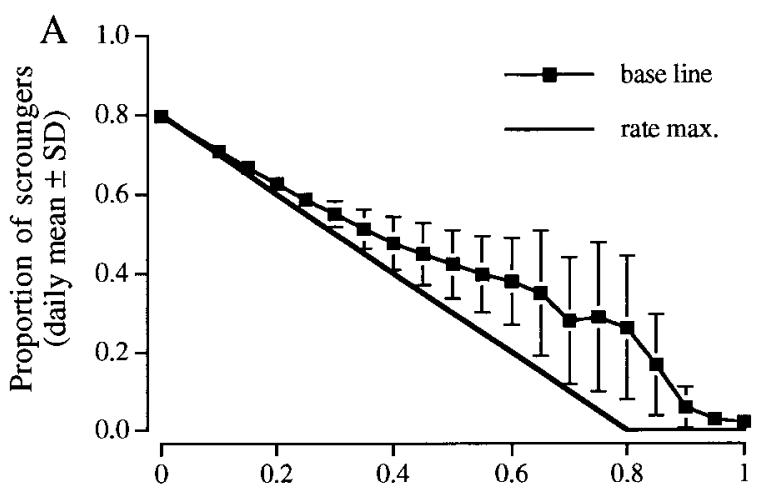

Finder's share

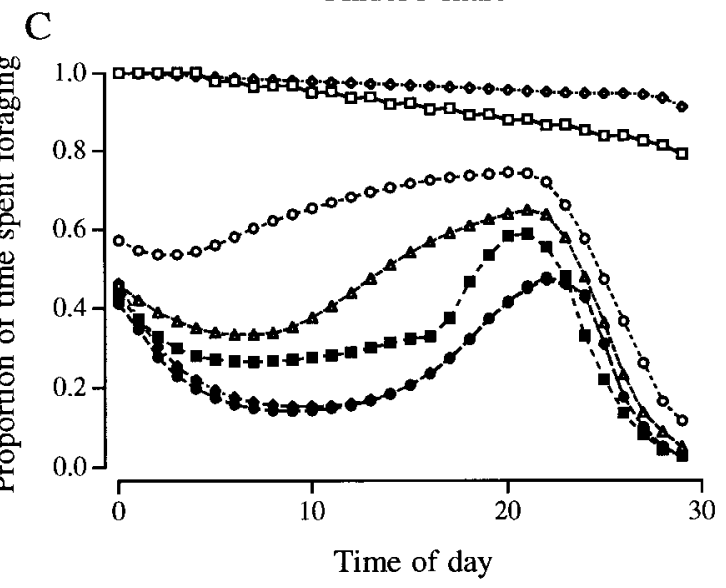

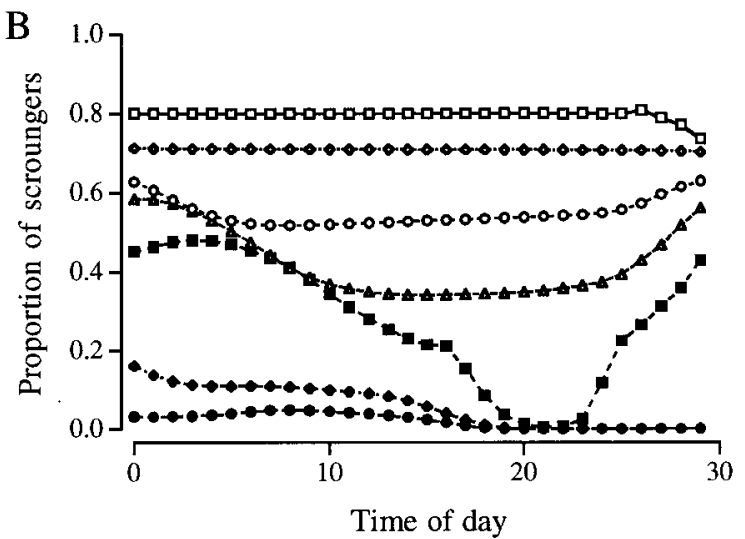

Finder's share

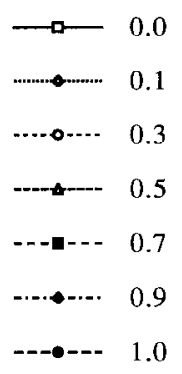

Figure 4: Effects of finder's share. A, Average proportion of scroungers ( \pm SD) over the course of the day drawn against the finder's share. Proportions identified by "rate max." were calculated by using Vickery et al.'s (1991) static model. B, Stable proportion of scrounger. C, Proportion of time spent foraging along the day at various values of finder's share.

decreasing FS (fig. 5). On the other hand, when food is highly clumped, the probability of daily survival reaches its maximum value at medium FS (fig. 5). Increasing the amount of food per patch has similar consequences. For small amounts of food the daily survival probability increases steadily with the FS (i.e., birds enjoy the highest survival when they do not share food with anyone; table 2). However, at large amounts of food per patch, intermediate FS provides higher survival than larger FS (table 2 ). The differences between maximum daily survival and daily survival with no food sharing $(F S=1.0)$ may appear small, but these differences could accumulate to around $5 \%$ after $15 \mathrm{~d}$.

\section{Predation Hazard}

Increasing predation hazard decreases the daily survival for all values of FS. On the other hand, varying predation hazard has no noticeable effect on either the average daily proportion of scrounger (fig. $6 \mathrm{~A}$ ) or the daily scrounger pattern, despite considerable variation in both the proportion of individuals engaged in foraging and the level of energy reserves (fig. 6B).

Recent studies (e.g., Ranta et al. 1998) hypothesize that the use of a scrounger foraging strategy could allow a player to detect an approaching predator sooner than if it played producer. This means that the scrounger strategy could provide its user with a lower predation hazard than the producer strategy. The increased detection ability of scrounging would be a by-product of having to survey the vicinity in order to find successful producers. We model this assumption by halving the predation hazard of individuals playing scrounger compared to those foraging as producers. Then we double predation hazard of the whole foraging group under this modified scenario to see whether it has any effect on the stable proportion of foragers playing scrounger. Reducing predation hazards of scrounging, not too surprisingly, increases the stable proportion of the scrounger strategy compared to the baseline case (fig. 6A). However, doubling predation hazards for the whole group 


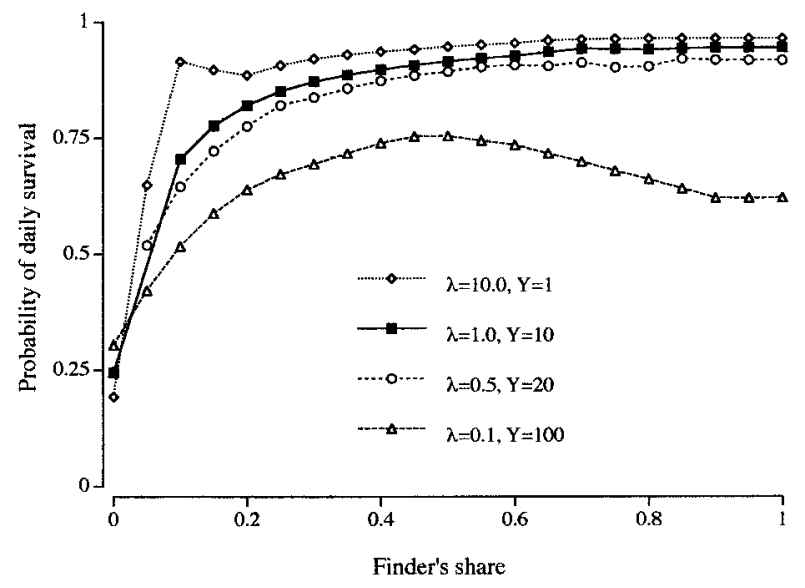

Figure 5: Probability of daily survival at different values of patchiness as the function of the finder's share. Patchiness was manipulated by varying both the food finding rate $(\lambda)$ and amount of food in a patch $(Y)$ so that their product remained constant. Increasing $Y$ with decreasing $\lambda$ means more aggregated food. Parameters are as baseline case except for amount of food and patch finding rate.

had no further effect on the stable proportion of scrounging (fig. 6A).

\section{Discussion}

Our state-dependent dynamic game model predicts (table 3 ) that the use of alternative foraging tactics, producer or scrounger, should depend on both the time of day and the animals' energetic reserves. Specifically, early in the day, low reserves promote a preference for the scrounger strategy, while the same condition late in the day favors the use of producer. As a consequence, individuals that play scrounger are expected to be lighter early but heavier late in the day than those opting to play producer. The stable proportion of individuals playing scrounger is predicted to change along the day; it is the highest around dawn and dusk and the lowest around midday. All of these predictions are the consequences of the risk-sensitive property of the foraging process; that is, the scrounger obtains small amounts of food in a reliable way while the producer can achieve either high or no food intake (fig. 1). Some empirical evidence from earlier work is consistent with the state-dependent dynamic model's prediction. Koops and Giraldeau (1996) report that food-deprived starlings first increased their proportional use of scrounger early in the day but then reduced it later in the day.

Around dawn in the winter, most birds have reserves that have dropped close to 0 following a night of metabolic expenditures without any feeding. So, at dawn, the birds face a high likelihood of starvation. To avoid starvation (i.e., letting reserves fall below the lower lethal boundary), the birds need only a small amount of food, but they need it reliably. Therefore, it is optimal with low reserves to chose the risk-averse scrounger option that allows the animals to gain some food almost certainly. In contrast to the standard risk sensitivity models (McNamara and Houston 1992b; McNamara 1996), however, the riskprone alternative (producer) is not eliminated from the behavioral repertoire at this time of the day because it is always advantageous to play producer when there are many scroungers in the group. This, however, is only optimal at higher reserves because of the high variation in producers' food intake. As a consequence of this process during early morning, one should observe many individuals with low reserves playing scrounger and a few individuals with high reserves playing producer.

The situation changes during midday. It becomes worth playing the risk-prone producer alternative more frequently because the strategy allows animals to have a higher chance of reaching high reserves and, therefore, switch to resting in order to avoid predation. At the same time, the punishment for preferring variation in food intake is low during this period of day because the animals have had time to gain energy and so their reserves are

Table 2: Probability of daily survival under different values of finder's share and amount of food $(Y)$

\begin{tabular}{lcccc}
\hline $\begin{array}{l}\text { Finder's } \\
\text { share }\end{array}$ & $Y=10$ & $Y=20$ & $Y=50$ & $Y=100$ \\
\hline .00 & .2446 & .8228 & .9516 & .9775 \\
.05 & - & .8788 & .9637 & .9837 \\
.10 & .7050 & .9088 & .9709 & .9878 \\
.15 & .7766 & .9266 & .9754 & .9901 \\
.20 & .8205 & - & .9781 & .9896 \\
.25 & .8510 & .9449 & .9815 & .9903 \\
.30 & .8711 & .9509 & .9813 & .9920 \\
.35 & .8858 & .9562 & .9812 & .9936 \\
.40 & .8974 & .9604 & .9829 & .9944 \\
.45 & .9067 & .9638 & .9870 & .9958 \\
.50 & .9147 & .9669 & .9899 & $.9963^{\mathrm{a}}$ \\
.55 & .9211 & .9690 & .9902 & .9960 \\
.60 & .9263 & .9724 & .9890 & .9960 \\
.65 & .9345 & .9745 & .9900 & .9960 \\
.70 & .9416 & .9760 & .9907 & .9960 \\
.75 & .9412 & $.9764^{\mathrm{a}}$ & $.9908^{\mathrm{a}}$ & .9959 \\
.80 & .9396 & .9760 & .9907 & .9959 \\
.85 & .9428 & .9757 & .9902 & .9955 \\
.90 & .9441 & - & .9896 & .9957 \\
.95 & .9440 & .9734 & .9887 & .9953 \\
1.00 & $.9443^{\mathrm{a}}$ & .9734 & .9872 & .9935 \\
\hline
\end{tabular}

Note: The minus sign marks cells where stable solution could not be reached with the baseline extent of error $(\delta)$.

${ }^{a}$ Maximum survival probabilities for the given amount of food. 

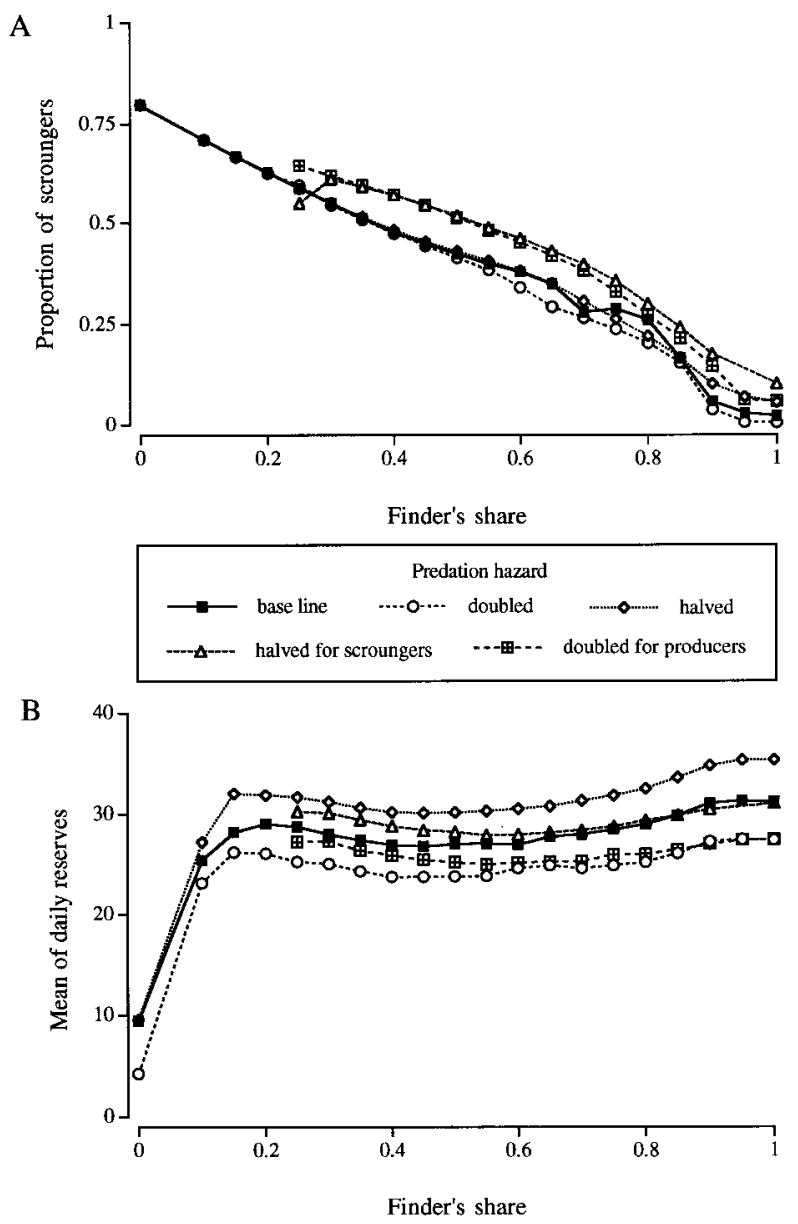

Figure 6: Effects of changing predation hazard. Changes in $(A)$ daily average proportion of scroungers and $(B)$ means of daily reserves with finder's share across different predation hazard. Predation hazard was manipulated by shifting both the base predation hazard (when $x=0$ ) and the rate of increase. This way predation hazard was doubled or halved at all values of state variable. Values of all other parameters are as in the baseline case.

already far from the lower lethal boundary; that is, the risk of starvation is much lower.

When dusk is approaching, it becomes important to reach or to keep a critical level of reserves that would insure overnight survival. Most of the animals following the optimal policy throughout the day are likely to be close to or at this required energy reserve. Therefore, most of these animals need only a small but reliable amount of food to reach or to maintain their critical reserve levels. Consequently they play it safe by resorting more to the scrounger strategy. However, those few individuals who are still far from the critical overnight reserve level will increase their chances of survival only by resorting more to the risk-prone alternative and play producer, a predic- tion that is in agreement with other risk-sensitivity models (McNamara and Houston 1992b).

Previous studies (e.g., Giraldeau et al. 1990, 1994; Vickery et al. 1991; Ranta et al. 1996) portrayed the presence of the scrounger strategy within groups as an inevitable cost of group living because scrounging decreases the mean intake of food for all members of a foraging group. In a stochastic, state-dependent, and dynamic world, however, the availability of the scrounger strategy may, instead, be seen as a benefit by providing an insurance against energetic shortfalls (Caraco and Giraldeau 1991; Koops and Giraldeau 1996). Our model supports this view that the availability of scrounging can be profitable because, assuming patches contain enough food, an individual's probability of daily survival was higher under conditions when the scrounger tactic was available (i.e., at medium values of FS) compared to cases where food sharing, and hence the scrounger strategy, was impossible $(\mathrm{FS}=1.0)$. This insurance provided by the scrounger strategy may explain why our model predicts more scroungers for high values of FS than previous models (e.g., Vickery et al. 1991) did. Note that we consider FS in our model as an inevitable constraint over which individuals have no control. If individuals can alter FS (e.g., through dominance relationships) things may change because a producer's interest may be to consume all of the patch alone (i.e., FS = 1.0) while scroungers would find it more profitable to share the whole patch $(\mathrm{FS}=0.0)$.

Scrounging can be called a risk-averse tactic in the sense that it decreases the chance of obtaining no food (fig. 1). As a consequence, one may expect that the availability of scrounging, that is, low FS, should increase individuals' probability of survival under all circumstances. Why does this only happen when food occurs in rare and/or rich patches? The explanation is that, while it is true that the scrounger strategy decreases the chance of finding no food (note, this effect is greater if food patches are rare), this outcome is counteracted by the consequent sharing with others. Sharing food decreases an individual's food intake so that the scrounger can only increase survival probability if the patches contain enough food to compensate for the loss through sharing.

Severe winter conditions are thought to be one of the main contributors to mortality in small passerine birds (McNamara and Houston 1987). Therefore, the increased survival probability provided by the scrounger alternative may have a great effect on a population's stability. It is surprising, however, that changes in predation hazard had no marked influence on the use of producer or scrounger strategies although, as hypothesized by Ranta et al. (1998), allowing the scrounger strategy to detect earlier the approaching predators does indeed lead to higher equilibrium proportions of scroungers within foraging groups. 
Table 3: Main testable predictions of the model

\begin{tabular}{ll}
\hline Prediction 1 & $\begin{array}{c}\text { Stable proportion of scrounger changes along the day: } \\
\text { Maximum proportion of scrounger around dawn and dusk; } \\
\text { Minimum proportion of scrounger around midday. }\end{array}$ \\
Prediction 2 & $\begin{array}{r}\text { Scroungers are lighter than producers during the first part of the day } \\
\text { but heavier later on. }\end{array}$ \\
Prediction 3 & Varying predation hazard does not change stable proportion of scrounger. \\
\hline
\end{tabular}

Whether the use of scrounging is compatible with predator detection, however, still needs to be addressed empirically.

An important feature of foraging groups, the dominance hierarchy, is still missing from our model. The effect of dominance hierarchy in a state-dependent dynamic world may be not so straightforward, but we can speculate on the basis of previous models. A recent static asymmetric producer-scrounger model that includes a social dominance hierarchy predicts that dominant individuals should prefer the scrounger strategy more than the subordinates (Barta and Giraldeau 1998). On the other hand, Clark and Ekman's (1995) state-dependent dynamic model predicts that dominants keep their reserves at a lower level than the subordinates. Combining these predictions with our own does not alter the predicted pattern very much because we predict that individuals with low reserves will use scrounger strategies more and that these will be the dominants who should keep low reserves and prefer the use of scrounging anyway. Empirical findings support these predictions (Baker et al. 1981; Rohwer and Ewald 1981; Czikeli 1983; Theimer 1987; Caraco et al. 1989; Clifton 1991). It would still be important, however, to distinguish the effects of dominance hierarchy from those of energetic state. To this end, future work should consider developing a state-dependent dynamic game model that includes dominance hierarchy.

In the current state-dependent dynamic model, we considered FS as a constraint: a parameter that individuals could not change. This approach, however, ignores the possibility that individuals could control FS by choosing to forage alone and hence to share food with no one. Individuals may choose to forage alone when food sharing does not provide an option that increases their survival probability. Our model identifies the conditions under which this would be the case as the availability of evenly distributed patches, each offering only small amounts of food. This argument is, of course, based on the assumption that the foraging gains from resorting to solitary foraging are sufficient to compensate the loss of safety from predators, which is seen as one of the major benefits of group living (Hamilton 1971; Caraco and Pulliam 1984; Clark and Mangel 1986; Elgar 1989; Székely et al. 1989, 1991).
Our model, including modifications such as the explicit modeling of predator attacks on foraging individuals (see, e.g., McNamara and Houston $1992 b$ for details), could cope with the decision of foraging alone versus within a group because it uses a common currency, daily survival, to compare the value of different behavioral actions. Such a future development could explicitly take into account the changes in predation hazard caused by switching from group to solitary foraging and by doing so would help integrate questions of group size and the use of scrounging that have to date remained distinct research traditions within social foraging.

To summarize, our state-dependent dynamic producerscrounger game provides testable predictions (table 3) about how time of day and animals' energetic reserves affect social foraging decisions. It further supports the counterintuitive argument that the availability of a scrounger foraging strategy can improve the daily survival of any average group member despite its selfish exploitative features. The model also indicates that more empirical work is needed to clarify the role of predator detection in this foraging context. Moreover, the model's development identified two important areas of future theoretical work: including the effect of dominance hierarchy and investigating the decision between foraging alone or in a group of exploiters.

\section{Acknowledgments}

This study was supported by Hungarian Scientific Research Fund (OTKA) grants (F16818 and T030434) to Z.B., a Natural Sciences and Engineering Research Council (Canada) research grant and Fonds pour la Formation de Chercheurs et l'Aide a la Recherche (Québec) Equipe grant to L.-A.G. Z.B. held a NATO Science Fellowship while developing the model and a János Bolyai Research Fellowship (Hungary) while preparing the manuscript. We are grateful to S. Dall, A. Sih, and an anonymous referee for helpful comments. 


\section{APPENDIX}

\section{Model Specification}

\section{Time and Actions}

We considered the birds' behavior during $5 \mathrm{~d}$ and nights. Each day was divided into 30 time intervals; that is, $t=$ 0 is dawn and $t=30$ is dusk. Length of nights is not specified in the model. At times $t=0,1, \ldots, 29$, the bird chooses an available action. During the day, it can either rest, produce, or scrounge, while during the night it rests.

\section{State Variable}

The bird's state on day $n$ is given by $X_{n}(t)$, which is the energy reserves. This variable takes nonnegative integer values. The bird dies if $X_{n}(t)=0$ because of starvation. Let the bird have reserves $i \geq 1$ at time $t$. Suppose it finds $E$ energy of food and uses metabolic energy $D$ between $t$ and $t+1$. Define $x=\operatorname{chop}\left(i+E-D, 0, x_{\max }\right)$, where $x_{\max }$ is the upper limit of reserves (energy that would raise reserves above this is lost); $\operatorname{chop}(y, a, b)$ (Clark and Mangel 1988) is defined as

$$
\operatorname{chop}(y, a, b)= \begin{cases}b & \text { if } y>b \\ y & \text { if } a \leq y \leq b \\ a & \text { if } y<a\end{cases}
$$

Since energy reserves take integer values, we define $[x]$ to be the integer part of $x$ and assume that reserves at $t+1$ are $[x]$ with probability $[x]+1-x$ and are $[x]+$ 1 with probability $x-[x]$ (Houston and McNamara $1988 a)$.

\section{Daylight Metabolism}

A bird that has reserves $i$ at time $t$ and behaves according to action $a$ between $t$ and $t+1$ expends $D_{a}=d 0_{a}+$ $d 2_{a} i^{2}$ amount of energy during this time interval. Action $a$ can be either resting, producing, or scrounging.

\section{Overnight Metabolism}

For overnight metabolism, $X_{n+1}(0)=\max \left[0, X_{n}(30)-\right.$ $\left.D_{\text {night }}\right]$, where $D_{\text {night }}$ is a random variable that follows an approximate normal distribution with mean $\mu_{\text {night }}$ and standard deviation $\sigma_{\text {night }}$.

\section{Foraging}

A bird using foraging tactic $f$ (producer or scrounger) finds $E_{f}=k_{f} F_{f}$ amount of food during a time interval. The amount of food a bird obtains from a discovered patch is $F_{f}$, while $k_{f}$ is the number of discovered patches between $t$ and $t+1$. The number of discovered patches $k_{f}$ follows a Poisson distribution with $\lambda_{f}$. A producer obtains $F_{\mathrm{P}}=$ $a+(Y-a) / n$ amount of food from a patch, while a scrounger gets $F_{\mathrm{S}}=(Y-a) / n$ amount. All patches contain $Y$ amount of food, from which $a$ (the finder's advantage; Vickery et al. 1991) is consumed by the patch-discoverer producer alone while $Y-a$ is shared equally among the producer and the arriving scroungers (i.e., $n=1+N_{\mathrm{S}}$ ). Finder's share is defined as $\mathrm{FS}=a / Y$. Producers find patches with rate $\lambda_{\mathrm{P}}$, while producer-monitoring scroungers find them with $\lambda_{\mathrm{S}}=\lambda_{\mathrm{P}} N\left[1-p_{\mathrm{S}}(t)\right]$ (Clark and Mangel 1986), where $N$ is the size of the foraging group and $p_{\mathrm{S}}(t)$ is the proportion of scroungers at time $t$.

\section{Predation Hazard}

Resting birds both during night and day are safe from predation. A foraging bird that has reserves $i$ at $t$ and use tactic $f$ is killed between $t$ and $t+1$ by a predator with probability $m_{f}=m 0_{f}+m 2_{f} i^{2}$.

\section{The Dynamic Programming Function}

Birds receive the terminal reward $R(x)=1$ if they survive the fifth night (i.e., $X_{6}(0)>0$ ), otherwise they receive $R(0)=0$. The optimal policy that maximizes expected reward is found by dynamic programming extended by the "errors in decision making" approach (Houston and McNamara 1987, 1988a; McNamara et al. 1997). First, we assume that the population follows an arbitrary policy, and so $p_{\mathrm{S}}(t)$ is an arbitrary function of $t$. Given this condition, the best response of a mutant can be determined by backward induction. A mutant that is in state $y$ at time $t+1$ and follows the optimal policy from time $t+1$ onward has the reproductive value $W(y, t+1) ; W(y, T)=$ $R(y)$. Let $W_{a}(x, t)$ be the mutant's reproductive value given that the mutant has reserves $x$ at time $t$ and it chooses action $a$ at $t$ and then follows the optimal policy from $t+1$ onward. The reproductive value for a resting mutant is $W_{r}(x, t)=W\left[\max \left(x-D_{r}, 0\right), t+1\right]$, while, for a mutant foraging with tactic $f$, it is 


$$
\begin{aligned}
W_{f}(x, t)=\left(1-m_{f}\right) \sum_{k=0} p_{k}^{(f)} W[\operatorname{chop}( & x-D_{f} \\
& \left.\left.+E_{f}, 0, x_{\max }\right), t+1\right] .
\end{aligned}
$$

The probability of finding $k=0,1, \ldots$ food patches between $t$ and $t+1$ according to a Poisson distribution with parameter $\lambda_{f}$ is

$$
p_{k}^{(f)}=\exp \left(-\lambda_{f}\right) \frac{\lambda_{f}^{k}}{k !}
$$

Because of minimizing computational effort, the sum over $k$ ran until either $k=50$ or $1-\Sigma p_{k}^{(f)}<10^{-5}$, whichever occurred first. In both cases, $\Sigma p_{k}^{(f)}$ was normalized to sum up to 1 . Days were linked by the approximation of the equation

$$
W_{n}(x, T)=\int W_{n+1}(x-y, 0) f(y) d y
$$

where $f(y)$ is the density function for $D_{\text {night }}$.

From the reproductive values for each action $W_{a}(x, t)$, one can determine $W(x, t)$. To calculate $W(x, t)$, we follow McNamara et al.'s (1997) "errors in decision making" approach throughout. First, we calculate the cost of choosing an action

$$
C_{a}(x, t)=\max \left[W_{a}(x, t)\right]-W_{a}(x, t),
$$

then assign a weight to each action a

$$
B_{a}(x, t)=H_{\delta}\left[C_{a}(x, t)\right]
$$

using the error function $H_{\delta}(u)=\exp (-u / \delta)$. After having the weights, we calculate the probability that action $a$ will be chosen by the mutant $p_{a}(x, t)=B_{a}(x, t) / \Sigma B_{a}(x, t)$. These probabilities give the optimal policy at $x, t$. Having the optimal policy, we calculate $W(x, t)=\Sigma p_{a}(x, t) W_{a}(x, t)$. After calculating $W(x, t)$, the whole procedure can be repeated to determine $W(x, t-1)$ until $t=0$, and so the mutant's optimal policy can be determined for the entire period.

After calculating the mutant optimal policy, we replace the resident policy partly by the new mutant policy then calculate the distribution of reserves along the days with this new policy by the standard forward iteration technique (Mangel and Clark 1988; McNamara and Houston 1990). The extent of replacement was given by the replacement factor $(\mathrm{rf}=0.2)$, and the new policy $p_{a}^{N}(x, t)$ was determined as $p_{a}^{N}(x, t)=(1-\mathrm{rf}) p_{a}^{\prime}(x, t)+\operatorname{rf}_{a}(x, t) \quad$ where $p^{\prime}(x, t)$ is the resident policy. In the forward iteration, we used the policies derived by the backward induction for the first day to insure independence on terminal condition. Having the distribution of reserves and the optimal policy, we calculate the new proportion of scroungers, $p_{\mathrm{s}}(t)$, and then repeat the whole cycle of backward induction and forward iteration again. In backward induction, we used the proportion of scroungers calculated by forward iteration for the fifth day to eliminate dependencies on initial reserve distribution. The repetition of backward induction and forward iteration continued either until 5,000 repetitions or until the average difference between the mutant and the resident policy decreased below 0.00001, whichever occurred first. The reached policy, however, was consider as evolutionarily stable dynamic policy only in the second case. The difference between policies was calculated as

$$
\sqrt{\sum_{a}\left[p_{a}(x, t)-p_{a}^{\prime}(x, t)\right]^{2}}
$$

and was averaged across all values of $x$ and $t$. The optimal policy of the previous repetition is $p_{a}^{\prime}(x, t)$. The source code of the model is available from the authors upon request.

\section{Literature Cited}

Baker, M. C., C. S. Belcher, L. C. Deutsch, G. L. Sherman, and D. B. Thompson. 1981. Foraging success in junco flocks and the effects of social hierarchy. Animal Behaviour 29:137-142.

Barnard, C. J., and R. M. Sibly. 1981. Producers and scroungers: a general model and its application to captive flocks of house sparrows. Animal Behaviour 29: 543-550.

Barnard, C. J., and D. B. A. Thompson. 1985. Gulls and plovers: the ecology of mixed species feeding groups. Croom Helm, London.

Barta, Z., and L.-A. Giraldeau. 1998. The effect of dominance hierarchy on the use of alternative foraging tactics: a phenotype-limited producing-scrounging game. Behavioural Ecology and Sociobiology 42:217-223.

Caraco, T., and L.-A. Giraldeau. 1991. Social foraging: producing and scrounging in a stochastic environment. Journal of Theoretical Biology 153:559-583.

Caraco, T., and H. R. Pulliam. 1984. Sociality and survivorship in animals exposed to predation. Pages 279-309 in P. W. Price, C. N. Slobodchikoff, and W. S. Gaud, eds. A new ecology: novel approaches to interactive systems. Wiley-Interscience, New York.

Caraco, T., C. Barkan, J. L. Beacham, L. Brisbin, S. Lima, A. Mohan, J. A. Newman, W. Webb, and M. L. Whithiam. 1989. Dominance and social foraging: a laboratory study. Animal Behaviour 38:41-58.

Clark, C. W., and J. Ekman. 1995. Dominant and sub- 
ordinate fattening strategies: a dynamic game. Oikos 72: 205-212.

Clark, C. W., and M. Mangel. 1986. The evolutionary advantages of group foraging. Theoretical Population Biology 3:45-75.

Clifton, K. E. 1991. Subordinate group members act as food finders within stripped parrotfish territories. Journal of Experimental Marine Biology Ecology 145: 141-148.

Cuthill, I. C., and A. I. Houston. 1997. Managing time and energy. Pages 97-120 in J. R. Krebs and N. B. Davies, eds. Behavioural ecology: an evolutionary approach. Blackwell Science, Oxford.

Czikeli, H. 1983. Agonistic interactions within a winter flock of slate-coloured juncos (Junco hyemalis) evidence for the dominants' strategy. Zeitschrift für Tierpsychologie 61:61-66.

Dall, S. R. X., and M. S. Witter. 1998. Feeding interruptions, diurnal mass changes and daily routines of behaviour in the zebra finch. Animal Behaviour 55: 715-725.

Elgar, M. A. 1989. Predator vigilance and group size in mammals and birds: a critical review of the empirical evidence. Biological Review 64:13-33.

Giraldeau, L.-A., and G. Beauchamp. 1999. Food exploitation: searching for the optimal joining policy. Trends in Ecology \& Evolution 14:102-106.

Giraldeau, L.-A., J. A. Hogan, and M. J. Clinchy. 1990. The payoffs to producing and scrounging: what happens when patches are divisible? Ethology 85:132-146.

Giraldeau, L.-A., C. Soos, and G. Beauchamp. 1994. A test of the producer-scrounger foraging game in captive flocks of spice finches, Lonchura punctulata. Behavior Ecology and Sociobiology 34:251-256.

Hamilton, W. D. 1971. Geometry of the selfish herd. Journal of Theoretical Biology 31:295-311.

Houston, A. I., and J. M. McNamara. 1987. Singing to attract a mate: a stochastic dynamic game. Journal of Theoretical Biology 129:57-68.

- 1988a. Fighting for food: a dynamic version of the hawk-dove game. Evolutionary Ecology 2:51-64.

-1988b. A framework for the functional analysis of behaviour. Behavioural and Brain Sciences 11: 117-163.

- 1999. Models of adaptive behaviour: an approach based on state. Cambridge University Press, Cambridge. Houston, A. I., C. W. Clark, J. M. McNamara, and M. Mangel. 1988. Dynamic models in behavioural and evolutionary ecology. Nature (London) 332:29-34.

Houston, A. I., J. M. McNamara, and J. M. C. Hutchinson. 1993. General results concerning the trade-off between gaining energy and avoiding predation. Philosophical
Transactions of Royal Society London B, Biological Sciences 341:375-397.

Koops, M. A., and L.-A. Giraldeau. 1996. Producerscrounger foraging games in starlings: a test of meanmaximizing and risk-minimizing foraging models. Animal Behaviour 51:773-783.

Mangel, M., and C. W. Clark. 1988. Dynamic modeling in behavioral ecology. Princeton University Press, Princeton, N.J.

Maynard Smith, J. 1982. Evolution and the theory of games. Cambridge University Press, Cambridge.

McNamara, J. M. 1996. Risk-prone behaviour under rules which have evolved in a changing environment. American Zoologist 36:484-495.

McNamara, J. M., and A. I. Houston. 1986. The common currency for behavioral decisions. American Naturalist 127:358-378.

-1987. Starvation and predation as factors limiting population size. Ecology 68:1515-1519.

- 1990. State-dependent ideal free distributions. Evolutionary Ecology 4:298-311.

. 1992a. Evolutionarily stable levels of vigilance as a function of group size. Animal Behaviour 43:641-658.

- 1992b. Risk-sensitive foraging: a review of the theory. Bulletin of Mathematical Biology 54:355-378.

McNamara, J. M., R. H. Mace, and A. I. Houston. 1987. Optimal daily routines of singing and foraging. Behavioral Ecology and Sociobiology 20:399-405.

McNamara, J. M., A. I. Houston, and S. L. Lima. 1994. Foraging routines of small birds in winter: a theoretical investigation. Journal of Avian Biology 25:287-302.

McNamara, J. M., J. N. Webb, E. J. Collins, T. Székely, and A. I. Houston. 1997. A general technique for computing evolutionarily stable strategies based on errors in decision-making. Journal of Theoretical Biology 189: 211-225.

Parker, G. A. 1984. The producer/scrounger model and its relevance to sexuality. Pages 127-153 in C. J. Barnard, ed. Producers and scroungers: strategies of exploitation and parasitism. Chapman \& Hall, New York.

Ranta, E., N. Peuhkuri, A. Laurila, H. Rita, and N. B. Metcalfe. 1996. Producers, scroungers and foraging group structure. Animal Behaviour 51:171-175.

Ranta, E., N. Peuhkuri, H. Hirvonen, and C. J. Barnard. 1998. Producers, scroungers and the price of a free meal. Animal Behaviour 55:737-744.

Rohwer, S., and P. W. Ewald. 1981. The cost of dominance and advantage of subordination in a badge signalling system. Evolution 35:441-454.

Sibly, R. M. 1984. Models of producer/scrounger relationships within and between species. Pages 267-287 in C. J. Barnard, ed. Producers and scroungers: strategies of exploitation and parasitism. Croom Helm, London. 
Székely, T., T. Szép, and T. Juhász. 1989. Mixed species flocking of tits (Parus spp.): a field experiment. Oecologia (Berlin) 78:490-495.

Székely, T., P. D. Sozou, and A. I. Houston. 1991. Flocking behaviour of passerines: a dynamic model for the nonreproductive season. Behavioral Ecology and Sociobiology 28:203-213.

Theimer, T. C. 1987. The effect of seed dispersion on the foraging success of dominant and subordinate darkeyed juncos, Junco hyemalis. Animal Behaviour 35: 1883-1890.

van der Veen, I. T. In press $a$. Daily routines and predator encounters in yellowhammers (Emberiza citrinella) in the field during winter. Ibis.

. In press $b$. Effects of predation risk on diurnal mass dynamics and foraging routines of yellowhammers (Emberiza citrinella). Behavior Ecology.

Vickery, W. L., L.-A. Giraldeau, J. J. Templeton, D. L. Kramer, and C. A. Chapman. 1991. Producers, scroungers, and group foraging. American Naturalist 137: 847-863.

Witter, M. S., and I. C. Cuthill. 1993. The ecological costs of avian fat storage. Philosophical Transactions Royal Society London B, Biological Sciences 340:73-92.

Witter, M. S., I. C. Cuthill, and R. H. C. Bonser. 1994. Experimental investigations of mass-dependent predation risk in the European starling, Sturnus vulgaris. Animal Behaviour 48:201-222.

Associate Editor: Andrew Sih 